\title{
Evaluation of HOPG mounting possibilities for multiplexing spectrometers
}

Felix Groitl ${ }^{\mathrm{a}, \mathrm{b}}$, Marek Bartkowiak ${ }^{\mathrm{c}}$, Ryan M. Bergmann ${ }^{\mathrm{d}}$, Jonas Okkels Birk $^{\mathrm{b}, \mathrm{e}}$, Marton Markób,f , Alex Bollhalder ${ }^{\mathrm{c}}$, Dieter Graf ${ }^{\mathrm{c}}$, Christof Niedermayer $^{\mathrm{b}}$, Christian Rüegg ${ }^{\mathrm{b}, \mathrm{g}}$, Henrik M. Rønnow ${ }^{\mathrm{a}, \mathrm{h}}$

${ }^{a}$ Laboratory for Quantum Magnetism, École Polytechnique Fédérale de Lausanne, 1015 Lausanne, Switzerland

${ }^{b}$ Laboratory for Neutron Scattering and Imaging, Paul Scherrer Institut, 5232 Villigen, Switzerland

${ }^{c}$ Laboratory for Scientific Developments and Novel Materials, Paul Scherrer Institut, 5232 Villigen, Switzerland

${ }^{d}$ Division Large Research Facilities, Paul Scherrer Institut, 5232 Villigen, Switzerland

${ }^{e}$ Department of Physics, Technical University of Denmark (DTU), 2800 Kgs. Lyngby, Denmark

${ }^{f}$ Wigner Research Centre for Physics, Neutron Spectroscopy Department, 1525 Budapest, Hungary

${ }^{g}$ Department of Quantum Matter Physics, University of Geneva, 1211 Geneva, Switzerland

${ }^{h}$ Niels Bohr Institute, University of Copenhagen, 2100 Copenhagen, Denmark

\begin{abstract}
Four different methods for mounting HOPG analyzer crystals on Si holders have been evaluated in the design process of the new multiplexing spectrometer CAMEA. Contrary to neutron optics used in standard spectrometers, the new instrument concept employs a series of analyzer segments behind each other where the neutrons have to pass through the bonding compound of the different analyzer crystals. The different methods, namely screws, shellac, indium soldering and clips, have been evaluated with regards to background, transmission, cooling, activation and handling. The results presented here will give valuable input for future CAMEA-type spectrometers currently planned and designed at various neutron sources.
\end{abstract}

Keywords: neutrons, neutron optics, neutron scattering, multiplexing, HOPG, neutron spectrometer 


\section{Introduction}

In neutron spectroscopy, e.g. on triple-axis spectrometers (TAS), Bragg reflection from crystals is the most common used method to select a well defined wavelength band from a white neutron beam [1]. For cold instruments, highly oriented pyrolytic graphite $(\mathrm{HOPG})$ is the material of choice for both monochromator and analyzer devices since it has a high reflectivity [2] and can be described as an ideally imperfect non-absorbing crystal. The HOPG crystals are usually mounted on flat metal or silicon holders, which by mechanical rotation can then be used as a focusing device (see e.g. [3]).

In the past decades, two 'standard techniques' for mounting the HOPG have been established, namely glue and screws. Special glues based on a mixture of beeswax and colophonium or 2-component epoxy glue have been used [4], e.g. at the ILL, France, or at the cold TAS FLEXX at HZB, Germany. Often these glues are mixed with a neutron absorber, e.g. $\mathrm{GdO}_{4}$ powder, to prevent unwanted background arising from scattering of the hydrogen content of the glue or the support material. Another glue material mainly used at PSI, Switzerland, and at some instruments at the MLZ, Germany, is shellac. The second attachment technique is screwing the HOPG crystals on the holder, as e.g. done at the MACS spectrometer [5], which automatically reduces the background. However, it entails certain other risks. First, a hole needs to be drilled into the HOPG crystal. This might result in a local deformation of the lattice and thus a worsening of the mosaic spread around the hole. Second, a certain amount of expensive crystal material is lost due to the drilling. If a spectrometer is at the end position of a neutron guide, the amount of fixing material behind the HOPG crystals of the monochromator, to a certain extent does not effect the downstream performance.

However, due to the excellent transmission properties of HOPG for longer wavelengths [6] there are several realizations where the monochromator of one instrument is placed upstream in the beam of a consecutive instrument, e.g. the RNR12 cold neutron guide at SINQ, PSI, where the instruments NARZISS, 
ORION, DMC and SANS-II (in downstream order) share one guide or at PELICAN, ANSTO, Australia [4]. In the latter case, the amount of material other than HOPG needs to be reduced to a minimum in order to minimize the number of neutrons scattered out of the beam of the following instruments. In the special case of PELICAN, even the single monochromator crystals are consecutively in front of each other. Here, an alternative fixation method was successfully implemented by using indium as a solder material [4] and realizing large HOPG crystal by soldering smaller pieces together. Since there are only 3 consecutive monochromator stages and the solder spots are small and thin, the rather large neutron absorption of the indium can be neglected. Furthermore, since a monochromator of a spectrometer is normally not changed often, the activation of the indium in the direct, white beam is likewise not a problem. The method was also successfully implemented at KOOKABURRA, ANSTO, Australia [7] by soldering the HOPG crystal of the pre-monochromator to a Si base plate. Recently, several instruments of a new generation of multiplexing spectrometers, which use a consecutive series of upwards scattering analyzer arcs to optimize the efficiency for in plane scattering, have been proposed. This concept has become know as Continuous Angle Multiple Energy Analysis (CAMEA) [8, 9]. Types of this backend are under construction at the cold TAS RITA-II $[10,11]$ at the PSI, Switzerland, [9] and in the design phase for the future ESS source [8] and the cold TAS PANDA [12-14] at the MLZ, Germany [15]. Furthermore, the CAMEA-type multiplexing backend MULTIFLEXX [3] has been commissioned [16] at the recently upgraded cold TAS FLEXX at HZB, Germany, [17, 18] after successful prototype measurements [19]. CAMEA uses a large amount of HOPG crystals mounted in series behind each other in the beam and has a rather open design, i.e, the detectors are sensitive to background arising from neighbored analyzers. It is thus necessary to evaluate existing and new fixation methods for this kind of spectrometer.

In the course of the design process of the CAMEA backend at PSI, different fixation methods have been investigated with regard to background, transmission, operation in vacuum and durability under cooling. Here, the results for 
90 Fig. 1 shows the design drawing of an angular segment for the CAMEA specthe evaluation of HOPG fixed with glue, screws, solder and mechanical clips are reported.

\section{Requirements}

The principal CAMEA instrument would consist of a series of focusing analyzer arcs where each arc is set to a fixed final energy. In reality, these arcs are modeled by angular modules where one module contains several analyzer segments which are placed behind each other to cover the different final energies. A detailed description of the CAMEA concept, the backend constructed at PSI and focusing conditions can be found in [9]. The horizontal opening angle of the angular module $\left(7.5^{\circ}\right)$ and the Bragg conditions of the analyzer segments determine the useful length of the individual HOPG crystals. For the analyzer closest to the sample $\left(E_{f}=3.2 \mathrm{meV}, 2 \Theta_{A}=97.8^{\circ}\right)$ the HOPG length is much smaller than for the analyzer furthest from the sample $\left(E_{f}=5 \mathrm{meV}\right.$, $\left.2 \Theta_{A}=74.2^{\circ}\right)$. Since frames of the front analyzer segments should not be in the beam in general and especially not in front of the rear crystals, it is not possible to mount the HOPG crystals directly to the frame for CAMEA (HOPG length first and last analyzer segment: $72 \mathrm{~mm}$ and $140 \mathrm{~mm}$, respectively). The option of extending the HOPG crystals more than necessary is discarded, since it is expensive, mechanically challenging and increases the background due to neutrons directly scattered into the shielding. Thus, additional supports are needed for the HOPG crystals. For CAMEA, Si was chosen as the holder material since it is stable and large single crystals, which have an excellent transmission for cold neutrons and produce almost no background, are available. The corresponding blades are cut out of standard double side polished $\left(\begin{array}{lll}1 & 0 & 0\end{array}\right)$ wafers with the $\left(\begin{array}{ll}0 & 1\end{array}\right.$ 0) orientation along the long axis. With this orientation the $\left(\begin{array}{lll}1 & 1 & 1\end{array}\right)$ reflection does not cause any spurious signals in the energy range used for CAMEA. The wafers are nearly perfect single crystals with a mosaicity in the range of a few arc seconds. 


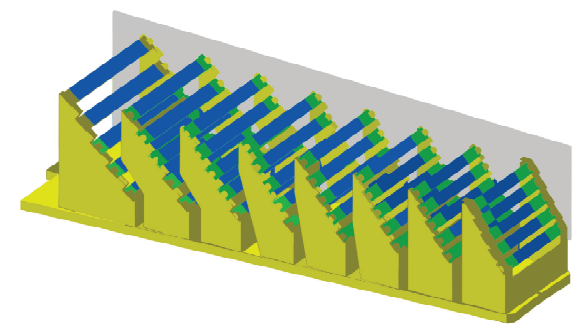

Figure 1: Design of the analyzer segments of one module. The HOPG crystals (blue) are mounted on Si blades (green), which are fixed within aluminum holders (yellow). Figure taken from $[9]$

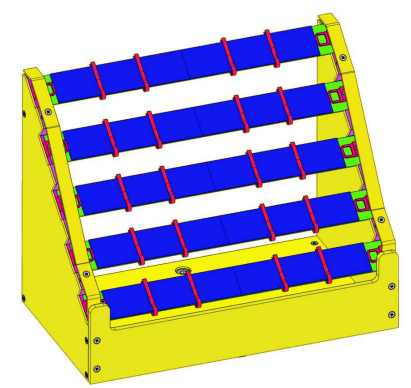

Figure 2: Detailed design drawing of the most outer analyzer segment $\left(E_{f}=5 \mathrm{meV}\right)$ as seen in Fig. 1. The total HOPG length of $140 \mathrm{~mm}$ is achieved by composing two $70 \mathrm{~mm}$ long crystals (blue, separation shown by black line). Here the crystals are fixed with the clip solution discussed in section 3.4.

${ }_{91}$ trometer currently constructed at PSI. Here, the HOPG (blue) is mounted on

92 Si blades (green), which are mounted in aluminum frames (yellow). A detailed

93 design drawing of the most outer analyzer segment (HOPG length of $140 \mathrm{~mm}$,

$\left.{ }_{94} E_{f}=5 \mathrm{meV}\right)$ is shown in Fig. 2. Here the HOPG crystals are fixed with the

95 clip solution discussed in section 3.4. The total HOPG length of $140 \mathrm{~mm}$ is

96 achieved by the composition of two $70 \mathrm{~mm}$ long HOPG crystals due to the fact,

97 that at the time of the design of CAMEA a maximum HOPG crystal length of

${ }_{98} 75 \mathrm{~mm}$ with the required homogeneity in mosaic spread was available. In fact,

99 such a composition is necessary for all analyzer segments except for the first one

100 (HOPG length of $72 \mathrm{~mm}, E_{f}=3.2 \mathrm{meV}$ ).

101

102 Contrary to the frames, the fixation compound used to mount the HOPG 


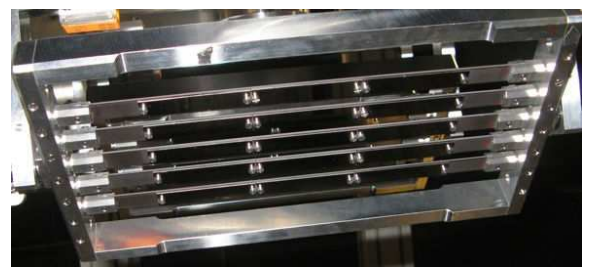

Figure 3: Analyzer frame of the CAMEA prototype. The frame holds Si wafers. The HOPG is fixed on the Si by aluminum screws. Picture taken from [21].

crystals on the Si blades is in the neutron beam. Thus, the classic and new fixation methods need to be evaluated taking several conditions into account:

- Absorption: The compound should have a low absorption cross section to avoid a reduction of the flux on consecutive analyzer segments

- Scattering: The compound should have a low scattering cross section to reduce additional background and beam attenuation

- Vacuum compatibility: The analyzer will be mounted within a vacuum tank and thus the compound must be suitable accordingly

In the evaluation process, fixation by glue (shellac), screws, solder (indium) and mechanical clips was evaluated. For some of the compounds, the durability under cooling was tested. This is especially interesting for future instruments where the setups might get closer to back-scattering geometry. Here, a cooling of the analyzer crystals can help to suppress the Lorentzian Bragg tails [20].

\section{Fixation methods}

\subsection{Screws}

Examples for the fixation of HOPG analyzer crystal with screws are the MACS spectrometer, NIST, and the CAMEA prototype at SINQ, PSI [21, 22]. At the latter, two to three crystals are mounted on long Si blade (see Fig. 3) using aluminum screws. The advantage of this method at room temperature is the easy mounting of the crystal and the replaceability of the crystals. However, a hole or a notch needs to be milled into the crystal. By doing this the 
crystal mosaicity around the hole/notch might be deteriorated. This has been observed at the crystals used at the CAMEA prototype. Measurements were performed on the residual stress diffractometer POLDI, SINQ [23, 24]. Here, small areas of the HOPG crystal (sample angle fixed, spot size $1.5 \times 1.5 \mathrm{~mm}^{2}$ ) are investigated using the white beam in combination with the radial detector. This allows for a fast, detailed scanning of the crystal. A detailed description of the used setup and exemplary data is presented in [21]. Additional measurements were performed on the multi purpose beamline BOA, SINQ, [25] using the energy-selective neutron imaging method [26]. Here, the sample angle was fixed to $38.8^{\circ}$ to diffract a wavelength of $4.2 \AA$ - a wavelength in the typical CAMEA working range. The incident energy was scanned from 4 Åto 4.4 Ain 0.02 Åsteps. A $100 \mu \mathrm{m}$ thick ${ }^{6}$ Li-based scintillator screen was used in combination with the Andor Ikon-M CCD camera [25] (1024×1024 pixel $^{2}$ focused on a $109 \times 109 \mathrm{~mm}^{2}$ field-of-view) to record neutrons transmitted through the sample. The transmitted intensity as a function of incident energy gives a direct measure of the mosaicity variations with a spatial resolution of $\approx 100 \mu \mathrm{m}$. A detailed description of the method itself and the setup can be found in [26]. Fig. 4 shows the map of the fitted Gaussians standard deviation $\sigma_{\lambda}$ of three crystals as measured [26]. It is clearly visible that the mosaic spread of the crystal around the notches is slightly deteriorated. One option to avoid this effect would be to make precise milling masks, which at the same time press on the crystal such that the milling effect on the edge of the hole/notch is minimized. For a larger spectrometer with a large analyzer area, this method would drastically increase the amount of work. Another drawback is the loss of expensive analyzer material due to the milling.

In order to investigate the compatibility for cooling, a frame from the CAMEA prototype (see Fig. 3) equipped with one Si blade and two HOPG crystals was mounted on a base plate within a closed cycle CTI and cooled down to $10 \mathrm{~K}$. To monitor the cooling process thermometers were placed on the bottom of the base plate, on the frame and on the HOPG in the middle of the frame. The results are shown in Fig. 5, top. The thermal contact between the holder and 


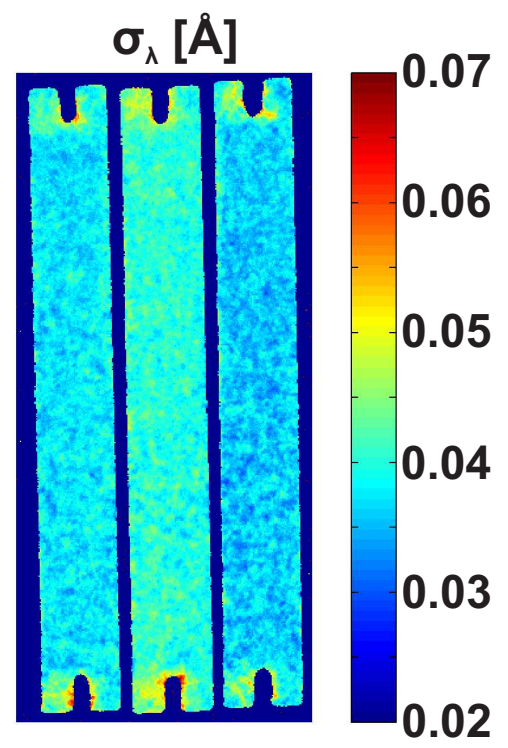

Figure 4: Three crystals from the CAMEA prototype were measured using the energy-selective neutron imaging method. Here, the transmitted neutron intensity at a fixed sample angle is measured as a function of incident energy. The transmitted intensity gives a direct measure of the mosaicity. The fitted Gaussians standard deviation $\sigma_{\lambda}$ is shown. It is clearly visible, that the mosaic spread is slightly deteriorated around the notches. Plot taken from [26], Fig. 5.13 therein. 

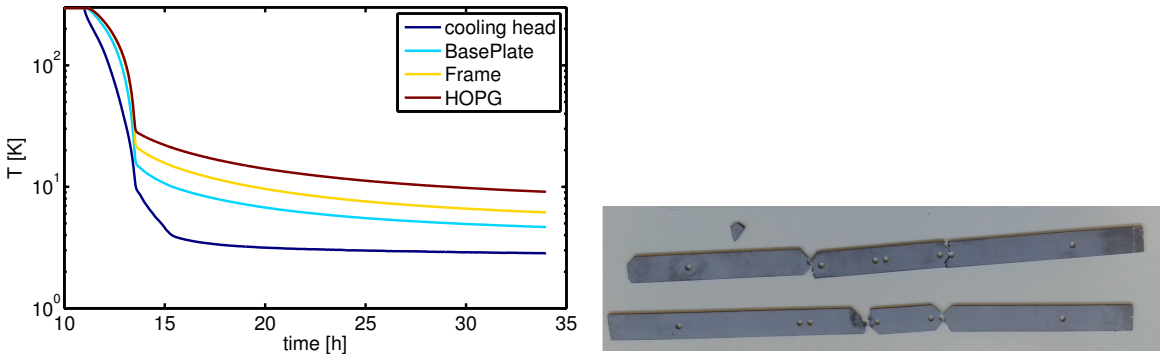

Figure 5: Top: Test setup temperatures as a function of cooling time. The thermal contact between the different components was found to be good and the the HOPG crystal reached a temperature of $10 \mathrm{~K}$ after $24 \mathrm{~h}$. Bottom: Si holders broken during the cooling tests. The holes in the Si clearly weaken the wafer.

the crystal was found to be good and the temperature is reached within $24 \mathrm{~h}$.

However, the mounting of the Si blade within a frame could cause additional problems. On one side, a good thermal contact between frame and Si is necessary to ensure the cooling. On the other side, the Si holders can not be clamped tight into the frame, since the Si blade is weakened by the milled holes, which work as a predetermined breaking point, and different thermal expansion coefficients from the frame material can easily break the blade (see Fig. 5, bottom). Estimations on the background arising from additional aluminum in the neutron beam are discussed in section 4 . Since aluminum is the only compound necessary for this method, a successful operation in vacuum is not affected.

\subsection{Shellac}

Shellac is a resinous substance that, dissolved in ethanol, can be used as a adhesive varnish. This method has been used at the PSI, Switzerland, and the FRM II, Germany, to mount HOPG monochromator or analyzer crystals. The fixation procedure is the following: The liquid shellac is applied to the holder and subsequently the HOPG crystal is placed on the holder. The stack is then weighted down with a small weight and the glue is hardened at $90^{\circ} \mathrm{C}$ in the oven for several hours. The longer the hardening process takes, the better the degassing of the glue. If the hardening process is too short, the adhesive might still be too elastic, resulting in a later shift from the requested position. Furthermore, for use in vacuum, the residual degassing might worsen the vacuum. 
For test measurements, HOPG crystals from the CAMEA prototype were glued on Si holders using shellac. It turned out that it is very difficult to apply a homogeneous, thin film of adhesive such that the HOPG surface is covered with glue and no excess glue is pressed sideways forming thicker beads. Such beads would need to be avoided in the CAMEA spectrometer, since they unnecessarily increase the amount of unwanted material in the neutron beam. Furthermore, it proved difficult to keep the HOPG in place before starting the hardening process, since the HOPG is almost free floating on the adhesive film. Due to the disadvantages in regards of handling and mounting glue was not chosen as the fixation method for CAMEA and no further investigation on different glues, e.g. hydrogen-free compounds, was performed.

In order to investigate potential effects on the Bragg tails and/or background, the $\left(\begin{array}{lll}0 & 0 & 2\end{array}\right)$ Bragg reflection of a HOPG crystal fixed with screws and a HOPG crystal glued with shellac was measured using the two-axes neutron diffractometer MORPHEUS at PSI. Sample rocking scans where performed using a neutron wavelength of $\lambda=4.67 \AA$. A sharp incoming beam $\left(\lambda_{F W H M} \approx 0.02 \AA\right)$ was obtained using the Si holder of the monochromator as the actual monochromatizing beam optics. The results are shown in Fig. 6. The blue data points (fixation with screw) where scaled by a factor of 0.845 to obtain the same peak intensity as for the black data points (fixation with shellac). The sum of a Gaussian and a Lorentzian was fitted to the data. No effect on the tails or on the background due to the different fixation methods is visible.

Additional measurements where performed to estimate the background arising from shellac. A thin aluminum can (diameter $10 \mathrm{~mm}$ ) was filled with shellac by repeatably applying a thin layer of liquid shellac and drying it in the oven. The incoherent scattering was measured with $\lambda=4.67 \AA$, corrected for the empty can measurement and compared to the signal from a vanadium rod of the same dimensions. The incoherent scattering measured is roughly a factor of 2.5 larger compared to that of vanadium. A comparison of the estimated background with that of aluminum is discussed in section 4.

Vacuum test were performed using one piece of HOPG fixed with shellac. After 


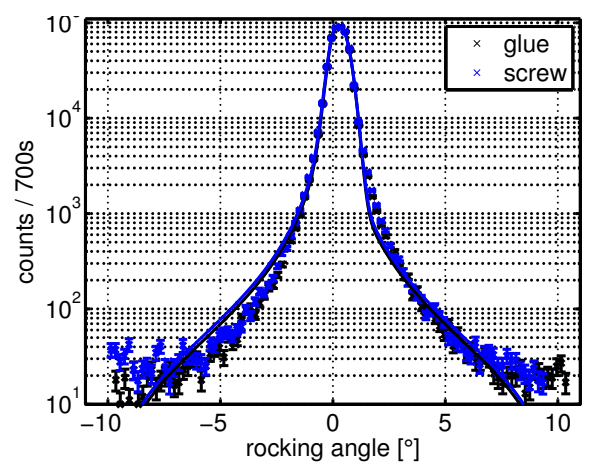

Figure 6: Rocking scans performed on the (lll 0 2 2) Bragg peak of HOPG fixed with screws (black) and shellac (blue, scaled with factor 0.845). No difference in background or peak shape a visible.

three days at a pressure of $2.1 \cdot 10^{-7}$ mbar no effect was noticeable. However, no long term test have been performed. Furthermore, since CAMEA employs $\approx 0.45 \mathrm{~m}^{2}$ of HOPG, a much larger amount of shellac in a larger vacuum vessel might worsen the vacuum quality.

\subsection{Indium}

The method of connecting HOPG crystals or fixing them to a Si holder using pure indium as solder in neutron beam optics was first used by A. K. Freund et al. $[4,7]$. As described in [4] indium wets sufficiently cleaned HOPG and Si surfaces well without an additional flux and it can easily be wiped off smooth surfaces.

The initial procedure is to apply indium solder spots manually on the HOPG crystal and the polished Si crystal by heating the crystals to a temperature of $170^{\circ} \mathrm{C}$ on a hot plate and spread the indium with a small spatula. The two components are then pressed together with a small weight while slowly cooling down. This should be done in a suitable mounting device to ensure a proper alignment of the HOPG crystal on the Si waver. Since CAMEA employs in total 630 pieces of HOPG crystals, this is a very time consuming procedure.

In addition there are up to two HOPG crystals per wafer, which could make alignment more difficult.

A second procedure was tested to allow for a better 'batch production'. The 
Si wafer was put into a template and small pieces $\left(2 \times 3 \mathrm{~mm}^{2}\right)$ of $50 \mu \mathrm{m}$ thick indium foil where laid on the wafer as solder spots. The HOPG crystals were put on top and the stack was weighted with a small piece of lead. The stack was heated up to $200^{\circ} \mathrm{C}$ for several hours and then slowly cooled down. The loosening of the connection showed that the indium melted very well. However, the bonding strength seemed less strong compared to the first procedure where the surface is manually wetted. In both cases, the connection is very sensitive to a bending of the Si wafer.

It has been shown that oxidation effects the wetting quality and the bonding strength for fluxless indium soldering [27]. If the soldering process is carried out under an inert environment, the indium wets the surface more homogeneously and the bonding strength is increased. Additional tests performed using the second procedure under inert environment showed good results. Further improvement of the bonding strength can be achieved by additionally preparing the Si wafers with thin sputtered In solder spots before the actual bonding process.

Indium provides an excellent thermal connection and thus is a good candidate for cooled analyzer crystals. Cycling the test samples between liquid nitrogen temperature and room temperature for several times did not show any effect on the solder connection. Since indium is the only compound used in this method, operation in vacuum is not affected.

Indium has a sizable absorption cross section and produces gamma radiation after activation. Since the solder spots are thin $(20-30 \mu \mathrm{m}[4])$, the calculated transmission losses for neutron wavelengths of $4-5 \AA$ are in the range of $4 \%$ per solder spot. In order to avoid a series of solder spot and thus a larger attenuation, the spots can be arranged accordingly.

Taking into account that each of the HOPG crystals of CAMEA is fixed with 4 solder spots (diameter $6 \mathrm{~mm}$, thickness $30 \mu \mathrm{m}$ ), a total mass of roughly $15.5 \mathrm{~g}$ of indium is within the neutron beam. Activation calculations were performed using CINDER'90 [28]. The activation was simulated using a flux spectrum from inside the neutron guide of the RITA-II beamline (calculated with MCNPX). 
This spectrum was renormalized to obtain a neutron flux of $1 \mathrm{e} 4 \mathrm{n} / \mathrm{cm}^{2} / \mathrm{s}$, which corresponds to a very conservative estimation of Bragg peak intensity illuminating the spectrometer. This intensity is unrealistic for CAMEA, however, it is a realistic estimate for high flux instruments to be built at the ESS. The activation is saturated after a 30-day irradiation with an activity of $8.4 \mu \mathrm{Ci}$. The arising background on the detectors can easily be discriminated by threshold adjustment. After $24 \mathrm{~h}$ the activity has already dropped to $0.1 \%$. The major gamma emitters in the short term are In116, In116m1 and In116m2, which have half lives of about $14 \mathrm{~s}, 54 \mathrm{~m}$, and $2 \mathrm{~s}$, respectively. After a day, In114 and $1 n 114 \mathrm{~m} 1$ are the main gamma emitters, and have half lives of about $72 \mathrm{~s}$ and $49 \mathrm{~d}$, respectively [28]. For CAMEA, the activation would not cause any problems and/or any additional instrument delay times in case of maintenance work. However, for spectrometers at the future high flux European Spallation Source (ESS) this might cause a problem and needs to be taken into account.

\subsection{Clips}

The second pure mechanical option investigated is fixation with clips. These clips are made out of Anticorodal AC110, a stiffer aluminum alloy, and can easily be cut by wire cutting. A 3D design drawing of such a clip is shown in Fig. 7. The height of the clip and the stopper are chosen such that the HOPG crystal and the Si wafer cannot slide out. The width of $2 \mathrm{~mm}$ in combination with the stamp at the end of the clip ensures that the HOPG does not tilt (when mounted with two clips). The length of the clip can easily be adjusted to the width of the analyzer crystals. In the case of CAMEA, the analyzer widths vary between $12 \mathrm{~mm}$ and $16 \mathrm{~mm}$. Pressure is applied at three contact points where the prebending of the clips is chosen such that a compression force of $59 \mathrm{mN}$ is applied. This has been proven to be sufficient to keep HOPG crystals in place even during vibrations or after small shocks. The clips are designed such, that they can be easily mounted from the side by slightly opening them and carefully sliding the over the crystals. This likewise allows for a nondestructive dismantling without breaking any connections and/or necessary cleaning. A 


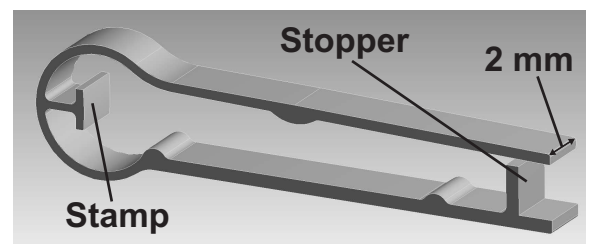

Figure 7: Clip solution to fix HOPG crystals on Si wavers.

lateral shift of the HOPG crystals can easily be prevented by adding very thin

Si wafers as fences.

The thermal contact between HOPG and $\mathrm{Si}$ is sufficient for cooling and the different thermal expansions of the different materials can be neglected on such a small scale taking into account the necessary tolerances for mounting the clips. Aluminum is the only compound used in this method, thus operation in vacuum is not affected. Estimations on the expected background due to the additional material in the beam are discussed in the next section.

\section{Background estimations for aluminum and shellac}

Since each crystal is hold by two aluminum clips and a total number of 1260 clips is needed resulting in an aluminum volume of $\approx 47 \mathrm{~cm}^{3}$. The same amount of aluminum screws (M2 x 4) including nuts would results in a total volume of $\sim 130 \mathrm{~cm}^{3}$. In this background estimation only the clip solution is considered. Assuming a $10 \mu \mathrm{m}$ thick shellac layer and a total area of HOPG of $0.45 \mathrm{~m}^{2}$ a total volume of shellac of $4.5 \mathrm{~cm}^{3}$ is required. In a first worst case scenario, one can compare the total cross sections (coherent and incoherent) assuming that the aluminum powder lines 'on average' reach the detectors. For the glue the total cross section would be dominated by incoherent scattering. As discussed above, that is a factor of $\approx 2.5$ of vanadium resulting in a cross section of $\approx 12.75$ barn $\left(\sigma_{V}=5.1\right.$ barn [29]). Aluminum would be dominated by coherent scattering $\left(\sigma_{c o h}=1.503\right.$ barn [29]). Thus, the cross section of shellac is a factor of 8.5 higher compared to aluminum. Since a $\approx 10$ times larger volume of the aluminum is needed, the worst case estimate of the background for both 
materials is comparable.

However, in reality it is too pessimistic to assume that the powder lines 'on average' reach the detectors. CAMEA employs a beryllium filter with a cut-off energy at $5 \mathrm{meV}$. Thus, the smallest $2 \Theta$ scattering angles are $118^{\circ}$ and $163^{\circ}$ for $\mathrm{Al}(111)$ and $\mathrm{Al}(200)$, respectively. Thus, for single scattering events, the scattering angles would be $>118^{\circ}$, which can not reach the detectors in the case of CAMEA. A minimum of two scattering events is required, which reduces the probability drastically. In comparison, the incoherent scattering arising from the shellac reaches the detectors in the amount corresponding to the solid angle view of the detectors from the analyzers. In the current design this is $\sim 0.04 \cdot \pi$ (taking into account the additional crosstalk shielding, otherwise it is $\left.\approx \frac{1}{10} \cdot 4 \pi\right)$. Therefore, it can be concluded, that the background expected from the aluminum clips is much less than the background due to scattering from shellac.

Taking into account the molecular weight $(\approx 586 \mathrm{~g} / \mathrm{mol})$ and density $\left(\approx 1.1 \mathrm{~g}^{2} \mathrm{~cm}^{3}\right)$ of shellac one can estimate the influence of a Bragg peak irradiating a row of analyzers. Assuming a Bragg peak flux of $10^{4} \mathrm{n} / \mathrm{s} / \mathrm{cm}^{2}$ and an angular coverage of $0.25^{\circ}$ results in an incoherent count rate of $\approx 50$ counts $/ \mathrm{min}$ arising from the shellac. This count rate is distributed in $4 \pi$ and as aforementioned $\approx 10 \%$ reach the detectors. The remaining rate of 5 counts $/ \mathrm{min}$ is distributed on 8 detector segments separated by a cross talk shielding. Thus, per analyzer segment a background rate of $\approx 0.6$ counts $/$ min can be expected which is comparable with typical background numbers.

For fixation with screws, the number of screws needed is the same as for clips.

The aluminum volume of an M2 screw (including nut and washers) is $\approx 120 \mathrm{~mm}^{3}$

and $\approx 3$ times larger than the volume of one clip $\left(\approx 38 \mathrm{~mm}^{3}\right)$. Thus, the background arising from a screw solution is expected to be larger compared to clips. However, due to the scattering conditions discussed above, it is still expected to be much lower than for shellac. 
Four different methods for mounting HOPG crystals on Si wavers have been 
R'Equip grant 206021-144972 and the Danish Natural Science Research Council through DANSCATT.

[1] I. S. Anderson, P. J. Brown, J. M. Carpenter, G. Lander, R. Pynn, J. M. Rowe, O. Schrpf, V. F. Sears, B. T. M. Willis, Neutron techniques, in: International Tables for Crystallography, Vol. C, IUCr, 2006, article 4.4, pp. $430-487$.

[2] T. Riste, K. Otnes, Oriented graphite as a neutron monochromator, Nuclear Instruments \& Methods 75 (2) (1969) 197-\&.

[3] K. Habicht, D. L. Quintero-Castro, R. Toft-Petersen, M. Kure, L. Maede, F. Groitl, M. D. Le, The upgraded cold neutron triple-axis spectrometer flexx - enhanced capabilities by new instrumental options, in: B. Frick, M. Koza, M. Boehm, H. Mutka (Eds.), QENS/WINS 2014 - 11TH INTERNATIONAL CONFERENCE ON QUASIELASTIC NEUTRON SCATTERING AND 6TH INTERNATIONAL WORKSHOP ON INELASTIC NEUTRON SPECTROMETERS, Vol. 83 of EPJ Web of Conferences, Inst LaueLangevin; ESS; FRMII; HZB; ILL; ISIS; JCNS; LLB; PSI, 2015, 11th International Conference on Quasielastic Neutron Scattering / 6th International Workshop on Inelastic Neutron Spectrometers (QENS/WINS), Autrans, FRANCE, MAY 11-16, 2014.

[4] A. K. Freund, D. H. Yu, Optimisation and fabrication of a composite pyrolytic graphite monochromator for the pelican instrument at the ansto opal reactor, NUCLEAR INSTRUMENTS \& METHODS IN PHYSICS RESEARCH SECTION A-ACCELERATORS SPECTROMETERS DETECTORS AND ASSOCIATED EQUIPMENT 634 (1) (2011) S75-S80, international Workshop on Neutron Optics, Grenoble, FRANCE, MAR 17-19, 2010.

[5] J. A. Rodriguez, D. M. Adler, P. C. Brand, C. Broholm, J. C. Cook, C. Brocker, R. Hammond, Z. Huang, P. Hundertmark, J. W. Lynn, N. C. Maliszewskyj, J. Moyer, J. Orndorff, D. Pierce, T. D. Pike, G. Scharfstein, 
S. A. Smee, R. Vilaseca, Macs - A new high intensity cold neutron spectrometer at nist, Measurement Science \& Technology 19 (3).

[6] D. Mildner, M. Arif, S. Werner, Neutron transmission through pyrolytic graphite monochromators, Journal of applied crystallography 34 (3) (2001) $258-262$.

[7] C. Rehm, L. de Campo, Kookaburra: The ultra-small-angle neutron scattering instrument at ansto, Neutron News 27 (2) (2016) 30-32.

[8] P. G. Freeman, J. O. Birk, M. Marko, M. Bertelsen, J. Larsen, N. B. Christensen, K. Lefmann, J. Jacobsen, C. Niedermayer, F. Juranyi, H. M. Ronnow, Camea ess - The continuous angle multi-energy analysis indirect geometry spectrometer for the European Spallation source, in: QENS/WINS 2014 - 11th International Conference on Quasielastic Neutron Scattering and 6th International Workshop on inelastic neutron spectrometers, Vol. 83 of EPJ Web of Conferences, EDP Sciences, 2015.

[9] F. Groitl, D. Graf, J. O. Birk, M. Marko, M. Bartkowiak, U. Filges, C. Niedermayer, C. Rueegg, H. M. Ronnow, Camea-a novel multiplexing analyzer for neutron spectroscopy, REVIEW OF SCIENTIFIC INSTRUMENTS $87(3)$.

[10] K. Lefmann, D. F. McMorrow, H. M. Rønnow, K. Nielsen, K. N. Clausen, B. Lake, G. Aeppli, Added flexibility in triple axis spectrometers: the two ritas at riso, Physica B 283 (4) (2000) 343-354.

[11] K. Lefmann, C. Niedermayer, A. B. Abrahamsen, C. R. H. Bahl, N. B. Christensen, H. S. Jacobsen, T. L. Larsen, P. Haefliger, U. Filges, H. M. Rønnow, Realizing the full potential of a RITA spectrometer, Physica B Condensed Matter 385-86 (2) (2006) 1083-1085.

[12] M. Loewenhaupt, N. Pyka, Design of the triple-axis spectrometer PANDA at the high-flux reactor FRM-II of Garching, Physica B-Condensed Matter 267 (1999) 336-340. 
[13] A. Schneidewind, P. Link, D. Etzdorf, R. Schedler, M. Rotter, M. Loewenhaupt, PANDA - first results from the cold three-axes spectrometer at FRM-II, Physica B-Condensed Matter 385-86 (2) (2006) 1089-1091.

[14] A. Schneidewind, P. Čermák, PANDA: Cold three axes spectrometer, Journal of large-scale research facilities 1 (A12).

[15] J. A. Lim, K. Siemensmeyer, P. Cermak, B. Lake, A. Schneidewind, D. S. Inosov, Bambus: a new inelastic multiplexed neutron spectrometer for panda, in: M. Zhitomirsky, P. DeReotier (Eds.), International Conference on Strongly Correlated Electron Systems 2014 (SCES2014), Vol. 592 of Journal of Physics Conference Series, IOP Publishing LTD, 2015.

[16] F. Groitl, R. Toft-Petersen, M. Kure, J. Lim, P. Cermak, S. Alimov, T. Wilpert, M. D. Le, D. Quintero-Castro, C. Niedermayer, A. Schneidewind, K. Habicht, Commissioning of multiflexx.

[17] M. D. Le, D. L. Quintero-Castro, R. Toft-Petersen, F. Groitl, M. Skoulatos, K. C. Rule, K. Habicht, Nuclear Instruments \& Methods in Physics Research section A - Accelerators Spectrometers Detectors and Associated Equipment 729 (2013) 220-226.

[18] M. D. Le, M. Skoulatos, D. L. Quintero-Castro, R. Toft-Petersen, F. Groitl, K. C. Rule, K. Habicht, Neutron News 25 (2014) 19-22.

[19] R. Toft-Petersen, F. Groitl, M. Kure, J. Lim, P. Cermak, S. Alimov, T. Wilpert, M. D. Le, D. Quintero-Castro, C. Niedermayer, A. Schneidewind, K. Habicht, Experimental characterization of a prototype secondary spectrometer for vertically scattering multiple energy analysis at cold-neutron triple axis spectrometers, Nuclear Instruments and Methods in Physics Research Section A: Accelerators, Spectrometers, Detectors and Associated Equipment 830 (2016) 338 - 344.

[20] M. T. F. Telling, K. H. Andersen, Spectroscopic characteristics of the osiris near-backscattering crystal analyser spectrometer on the isis pulsed neutron 
source, Phys. Chem. Chem. Phys. 7 (2005) 1255-1261.

URL http://dx.doi.org/10.1039/B413934H

[21] M. Markó, EPFL-report-197952, http:// infoscience.epfl.ch/ record/ 197952/ files/ prototye_report_march.pdf.

[22] M. Markó, F. Groitl, J. O. Birk, P. G. Freeman, K. Lefmann, N. B. Christensen, C. Niedermayer, F. Jurányi, A. Hansen, H. M. Rønnow, Prototype of the novel CAMEA concept - A backend for neutron spectrometers, to be published.

[23] U. Stuhr, H. Spitzer, J. Egger, A. Hofer, P. Rasmussen, D. Graf, A. Bollhalder, M. Schild, G. Bauer, W. Wagner, Time-of-flight diffraction with multiple frame overlap part ii: The strain scanner poldi at psi, NUCLEAR INSTRUMENTS \& METHODS IN PHYSICS RESEARCH SECTION A-ACCELERATORS SPECTROMETERS DETECTORS AND ASSOCIATED EQUIPMENT 545 (1-2) (2005) 330-338.

[24] U. Stuhr, M. Grosse, W. Wagner, The tof-strain scanner poldi with multiple frame overlap-concept and performance, MATERIALS SCIENCE AND ENGINEERING A-STRUCTURAL MATERIALS PROPERTIES MICROSTRUCTURE AND PROCESSING 437 (1, SI) (2006) 134-138, tMS Annual Meeting and Exhibition, San Francisco, CA, FEB 13-16, 2005.

[25] M. Morgano, S. Peetermans, E. Lehmann, T. Panzner, U. Filges, Neutron imaging options at the BOA beamline at Paul Scherrer Institut, Nuclear Instruments and Methods in Physics Research Section A: Accelerators, Spectrometers, Detectors and Associated Equipment 754 (0) (2014) 46 - 56.

[26] S. L. X. Peetermans, Energy-selective neutron imaging for materials science, Ph.D. thesis, EPFL (2015).

[27] J. Kim, H. Schoeller, J. Cho, S. Park, Effect of oxidation on indium solderability, Journal of Electronic Materials 37 (4) (2008) 483-489, symposium 

on Recent Developments in Semiconductor, Electro Optic and Radio Frequency Materials and Metrologies for Advanced Materials and Devices, Orlando, FL, FEB, 2007.

[28] W. B. Wilson, S. T. Cowell, T. R. England, A. C. Hayes, P. Moller, A manual for cinder'90 version 07.4 codes and data, Los Alamos National Laboratory report LA-UR-07- 8412 (2008).

[29] A.-J. Dianoux, G. Lander (Eds.), Neutron data booklet, Old City Publish487 ing, 2003. 